\title{
U.S. GEOLOGICAL SURVEY, DENVER, COLORADO RADIOCARBON DATES I*
}

\author{
L. J. SCHRODER, W. A. BEETEM, H. C. CLAASSEN, \\ and R. L. EMERSON
}
U.S. Geological Survey, Denver Federal Center, Lakewood, Colorado 80225

The U.S. Geological Survey, at the Denver Federal Center, Lakewood, Colorado assays $\mathrm{C}^{14}$ concentration of water samples using liquid scintillation techniques. The laboratory synthesizes benzene from precipitated barium carbonate using techniques described by Noakes, Kim, and Akers (1967). Three $\mathrm{ml}$ of the synthesized benzene is pipetted into a tared, low potassium- 40 glass vial. The sample is weighed and $1 \mathrm{ml}$ of a scintillation solution is added to the vial. The scintillation solution used is a mixture of $10 \mathrm{~g}$ PPO and $0.025 \mathrm{~g}$ dimethyl-POPOP scintillators in $250 \mathrm{ml}$ toluene. Calculations of dates are made with the radiocarbon half-life of 5568 years; plus or minus numbers quoted herein are the standard error for the counting of radioactive disintegrations.

Hydrologic studies are made by the U.S. Geological Survey in support of the nuclear testing program of the U.S. Atomic Energy Commission. Radiocarbon dates from samples of water obtained during these hydrologic studies are used to aid in hydrologic interpretations of the study areas. Data describing the wells and springs sampled were taken from records of the Nevada State Engineer and then field checked. Water samples were collected in a manner to minimize contamination by atmospheric $\mathrm{CO}_{2}$. Through the addition of barium chloride and a precipitation procedure, sufficient barium carbonate is obtained to yield at least $3 \mathrm{~g}$ carbon after synthesization.

All samples were collected by AEC Hydrology Projects, Water Resources Division, U.S. Geological Survey, Lakewood, Colorado, except for water samples from the State of New Mexico which were collected by U.S. Geological Survey, Water Resources Division, New Mexico District office. Alkalinity values were determined at time of sample collection.

The $\delta \mathrm{C}^{13}$ values reported in Tables 1 and 2 are based on Craig PDB limestone standard (Craig, 1957) equal to $0.00 \%$ and were determined by Teledyne Isotopes, Inc., Westwood Laboratories (Buckley and Willis, 1969). The total alkalinity as bicarbonate values reported in Tables 1 and 2 were determined using techniques described by Rainwater and Thatcher (1960). Conversion factor from gall $/ \mathrm{min}$ to $\mathrm{m}^{3} / \mathrm{min}$ was 0.0037854 .

\section{ACKNOWLEDGMENTS}

The measurement of $\mathrm{C}^{14}$ ages was financed through the Nevada Operations Office, U.S. Atomic Energy Commission.

\footnotetext{
* Publication authorized by the Director, U.S. Geological Survey.
} 
TABLE 1.

Summary of $\mathrm{C}^{14}, \delta \mathrm{C}^{13}$, and alkalinity for water sources in Nevada

\begin{tabular}{ccccc}
\hline $\begin{array}{c}\text { Sample } \\
\text { no. }\end{array}$ & Date & $\begin{array}{c}\text { Radiocarbon } \\
(\% \text { NBS })\end{array}$ & $\begin{array}{c}\delta \mathrm{C}^{13} \\
(\% \text { PDB })\end{array}$ & $\begin{array}{c}\text { Total alkalinity } \\
\text { as bicarbonate } \\
(\mathrm{mg} / \mathrm{l})\end{array}$ \\
\hline DE-670293 & $11 / 19 / 66$ & $5.1 \pm 0.8$ & -5.5 & 300 \\
DE-670294 & $11 / 19 / 66$ & $2.4 \pm 0.8$ & -6.9 & 300 \\
DE-670295 & $11 / 21 / 66$ & $11.1 \pm 1.4$ & -8.7 & 304 \\
DE-670296 & $11 / 20 / 66$ & $11.4 \pm 1.8$ & -11.0 & 310 \\
DE-670297 & $11 / 18 / 66$ & $8.0 \pm 1.6$ & -6.8 & 303 \\
DE-670298 & $10 / 20 / 66$ & $10.8 \pm 1.3$ & -4.8 & 278 \\
DE-670299 & $11 / 19 / 66$ & $2.9 \pm 1.4$ & -5.0 & 330 \\
DE-670300 & $11 / 21 / 66$ & $7.2 \pm 1.0$ & -7.4 & 304 \\
DE-670301 & $11 / 21 / 66$ & $3.7 \pm 1.6$ & -5.5 & 300 \\
DE-670302 & $11 / 20 / 66$ & $12.1 \pm 1.7$ & -7.9 & 302 \\
DE-670303 & $11 / 21 / 66$ & $3.0 \pm 1.3$ & -5.6 & 318 \\
DE-670341 & $12 / 09 / 66$ & $3.5 \pm 1.5$ & -6.8 & 310 \\
DE-670342 & $12 / 09 / 66$ & $14.8 \pm 2.1$ & -8.3 & 302 \\
DE-670343 & $12 / 10 / 66$ & $18.1 \pm 1.5$ & -9.9 & 234 \\
DE-670344 & $12 / 11 / 66$ & $17.8 \pm 1.6$ & -10.5 & 310 \\
DE-670345 & $12 / 12 / 66$ & $82.9 \pm 1.0$ & -11.6 & 307 \\
DE-670346 & $12 / 13 / 66$ & $20.4 \pm 1.3$ & - & 316 \\
DE-670347 & $12 / 14 / 66$ & $18.6 \pm 1.7$ & -11.1 & 268 \\
DE-670348 & $12 / 14 / 66$ & $80.3 \pm 0.9$ & - & 302 \\
DE-680076 & $07 / 07 / 67$ & $29.1 \pm 1.1$ & -8.5 & 139 \\
DE-680078 & $07 / 07 / 67$ & $9.2 \pm 1.0$ & -8.4 & 184 \\
DE-680080 & $07 / 05 / 67$ & $14.5 \pm 0.9$ & -5.5 & 207 \\
DE-680082 & $07 / 05 / 67$ & $26.2 \pm 1.0$ & -6.8 & 232 \\
DE-680089 & $07 / 05 / 67$ & $30.3 \pm 1.3$ & -5.2 & 183 \\
DE-680092 & $07 / 06 / 67$ & $27.6 \pm 1.2$ & -6.3 & 185 \\
DE-680097 & $07 / 04 / 67$ & $25.6 \pm 1.0$ & -6.8 & 228 \\
DE-680098 & $07 / 04 / 67$ & $37.6 \pm 1.4$ & -6.6 & 372 \\
DE-680101 & $07 / 03 / 67$ & $36.5 \pm 1.1$ & -7 & 185 \\
DE-680105 & $07 / 03 / 67$ & $34.1 \pm 0.8$ & -7.7 & 196 \\
DE-680106 & $07 / 03 / 67$ & $35.8 \pm 1.2$ & -7.2 & 212 \\
DE-680107 & $07 / 03 / 67$ & $65.1 \pm 1.4$ & -8.9 & 396 \\
DE-680583 & $11 / 16 / 67$ & $4.4 \pm 1.6$ & - & 258 \\
DE-680649 & $08 / 25 / 67$ & $51.6 \pm 1.5$ & -12.0 & 232 \\
DE-680905 & $02 / 14 / 68$ & $27.2 \pm 1.2$ & -7.6 & 189 \\
DE-680914 & $02 / 14 / 68$ & $7.3 \pm 0.9$ & -7.4 & 178 \\
DE-680960 & $03 / 04 / 68$ & $5.8 \pm 0.8$ & -5.5 & 580 \\
DE-680961 & $03 / 05 / 68$ & $5.9 \pm 0.7$ & - & 580 \\
DE-680962 & $03 / 06 / 68$ & $7.6 \pm 0.8$ & -6.9 & 580 \\
DE-680963 & $03 / 07 / 68$ & $8.7 \pm 0.8$ & -6.9 & 580 \\
DE-680964 & $03 / 08 / 68$ & $11.9 \pm 1.3$ & - & 580 \\
DE-681240 & $06 / 18 / 68$ & $12.7 \pm 2.0$ & - & 114 \\
\hline & & & &
\end{tabular}




\section{GROUNDWATER SAMPLES}

\section{DE-670293. Fairbanks Spring}

Sample coll. Nov. 19, 1966 from SW spring orifice, Fairbanks Spring, Amargosa Desert, Nye Co., Nevada $\left(36^{\circ} 29^{\prime} 30^{\prime \prime} \mathrm{N}\right.$ Lat, $116^{\circ} 20^{\prime} 30^{\prime \prime} \mathrm{W}$ Long) $17.7 \mathrm{~km}$ SSE of Lathrop Wells, Nevada. The total spring discharge was $6.5 \mathrm{~m}^{3} / \mathrm{min}$. at $27.0^{\circ} \mathrm{C}$ at alt. $694.9 \mathrm{~m}$ above $\mathrm{msl}$ from valley fill.

\section{DE-670294. Fairbanks Spring}

$30,000 \pm 2300$

Sample coll. Nov. 19, 1966 from NE spring orifice, Fairbanks Spring, Amargosa Desert, Nye Co., Nevada (36 29' $30^{\prime \prime}$ N Lat, $116^{\circ} 20^{\prime} 30^{\prime \prime} \mathrm{W}$ Long) $17.7 \mathrm{~km}$ SSE of Lathrop Wells, Nevada. The total spring discharge was $6.5 \mathrm{~m}^{3} / \mathrm{min}$. at $27.0^{\circ} \mathrm{C}$ at alt. $694.9 \mathrm{~m}$ above msl from valley fill.

\section{DE-670295. Point of Rocks Spring (King)}

$17,700 \pm 1000$

Sample coll. Nov. 21, 1966 from Point of Rocks Spring (King), Amargosa Desert, Nye Co., Nevada (36 24' 02" N Lat, $116^{\circ} 16^{\prime} 25^{\prime \prime} \mathrm{W}$ Long) $29.0 \mathrm{~km}$ SSE of Lathrop Wells, Nevada. The spring discharged $4.1 \mathrm{~m}^{3} / \mathrm{min}$. at $33.0^{\circ} \mathrm{C}$ from Paleozoic limestones at alt. $701.0 \mathrm{~m}$ above msl.

\section{DE-670296. Spring 17S/50-23bbc}

$$
17,400 \pm 1300
$$

Sample coll. Nov. 20, 1966 from Spring 17S/50-23bbc, Amargosa Desert, Nye Co., Nevada ( $36^{\circ} 27^{\prime} 36^{\prime \prime}$ N Lat, $116^{\circ} 21^{\prime} 14^{\prime \prime}$ W Long) $20.9 \mathrm{~km}$ SSE of Lathrop Wells, Nevada. Water issues from Paleozoic limestone at alt. $713 \mathrm{~m}$ above msl.

\section{DE-670297. Longstreet Spring}

$20,300 \pm 1200$

Sample coll. Nov. 18, 1966 from Longstreet Spring, Amargosa Desert, Nye Co., Nevada ( $36^{\circ} 28^{\prime} 04^{\prime \prime}$ N Lat, $116^{\circ} 19^{\prime} 31^{\prime \prime}$ W Long) $20.1 \mathrm{~km}$ SSE

TABLe 2.

Summary of $\mathbf{C}^{14}, \delta \mathbf{C}^{13}$, and alkalinity for water sources in New Mexico

\begin{tabular}{ccrcc}
\hline $\begin{array}{c}\text { Sample } \\
\text { no. }\end{array}$ & Date & $\begin{array}{c}\text { Radiocarbon } \\
(\% \mathrm{NBS})\end{array}$ & $\begin{array}{c}\delta \mathrm{C}^{13} \\
(\% \text { PDB })\end{array}$ & $\begin{array}{c}\text { Total alkalinity } \\
\text { as bicarbonate } \\
(\mathrm{mg} / \mathrm{l})\end{array}$ \\
\hline $\mathrm{DE}-680458$ & $09 / 04 / 67$ & $17.9 \pm 3.0$ & -12.5 & - \\
DE-680650 & $11 / 14 / 67$ & $47.9 \pm 1.6$ & -23.4 & - \\
DE-680651 & $11 / 20 / 67$ & $8.0 \pm 1.5$ & -10.3 & - \\
DE-680759 & $12 / 11 / 67$ & $22.5 \pm 1.6$ & -7.8 & - \\
DE-680782 & $10 / 10 / 67$ & $6.5 \pm 1.6$ & - & - \\
DE-680788 & $10 / 03 / 67$ & $29.1 \pm 2.4$ & - & - \\
\hline
\end{tabular}


of Lathrop Wells, Nevada. The spring discharged $3.9 \mathrm{~m}^{3} / \mathrm{min}$. at $27.0^{\circ} \mathrm{C}$ at alt. $701.0 \mathrm{~m}$ above msl from valley fill.

\section{DE-670298. Crystal Spring}

$17,900 \pm 1100$

Sample coll. Nov. 20, 1966 from Crystal Spring, Amargosa Desert, Nye Co., Nevada $\left(36^{\circ} 25^{\prime} 15^{\prime \prime} \mathrm{N}\right.$ Lat, $116^{\circ} 19^{\prime} 19^{\prime \prime}$ W Long) $25.7 \mathrm{~km}$ SSE of Lathrop Wells, Nevada. The spring discharged $10.7 \mathrm{~m}^{3} / \mathrm{min}$. at $33.0^{\circ} \mathrm{C}$ at alt. $670.6 \mathrm{~m}$ above $\mathrm{msl}$ from valley fill.

\section{DE-670299. Soda Ash Spring}

$28,400 \pm 4200$

Sample coll. Nov. 19, 1966 from Soda Ash Spring, Amargosa Desert, Nye Co., Nevada $\left(36^{\circ} 29^{\prime} 22^{\prime \prime} \mathrm{N}\right.$ Lat, $116^{\circ} 20^{\prime} 10^{\prime \prime} \mathrm{W}$ Long) $17.7 \mathrm{~km}$ SSE of Lathrop Wells, Nevada. The spring discharged $0.3 \mathrm{~m} / \mathrm{min}$. at $23.0^{\circ} \mathrm{C}$ at alt. $694.9 \mathrm{~m}$ above $\mathrm{msl}$ from valley fill.

\section{DE-670300. Point of Rocks Spring (small)}

$21,100 \pm 1200$

Sample coll. Nov. 21, 1966 from Point of Rocks Spring (small), Amargosa Desert, Nye Co., Nevada $\left(36^{\circ} 24^{\prime} 05^{\prime \prime} \mathrm{N}\right.$ Lat, $116^{\circ} 16^{\prime} 15^{\prime \prime} \mathrm{W}$ Long) $29.0 \mathrm{~km}$ SSE of Lathrop Wells, Nevada. The spring discharged 0.1 $\mathrm{m}^{3} / \mathrm{min}$. at $34.0^{\circ} \mathrm{C}$ at alt. $829.1 \mathrm{~m}$ above $\mathrm{msl}$ from Paleozoic limestone.

\section{DE-670301. Jack Rabbit Spring}

$>26,500 \pm 1400$

Sample coll. Nov. 21, 1966 from Jack Rabbit Spring, Amargosa Desert, Nye Co., Nevada (36 $26^{\circ} 23^{\prime \prime}$ N Lat, $116^{\circ} 16^{\prime} 41^{\prime \prime}$ W Long) $29.8 \mathrm{~km}$ SSE of Lathrop Wells, Nevada. The spring discharged $2.2 \mathrm{~m}^{3}$ min. at $28.0^{\circ} \mathrm{C}$ at alt. $691.9 \mathrm{~m}$ above msl from valley fill.

\section{DE-670302. Rogers Spring \\ $17,000 \pm 1200$}

Sample coll. Nov. 20, 1966 from Rogers Spring, Amargosa Desert, Nye Co., Nevada ( $36^{\circ} 28^{\prime} 48^{\prime \prime}$ N Lat, $116^{\circ} 19^{\prime} 32^{\prime \prime}$ W Long) $19.3 \mathrm{~km}$ SSE of Lathrop Wells, Nevada. The spring discharged $2.8 \mathrm{~m}^{3} / \mathrm{min}$. at $28.0^{\circ} \mathrm{C}$ at alt. $694.9 \mathrm{~m}$ above $\mathrm{msl}$ from valley fill.

\section{DE-670303. Big Spring (Deep Spring, $\quad 28,200 \pm 3000$ Ash Meadows Spring) \\ 26,250 B.c.}

Sample coll. Nov. 21, 1966 from Big Spring (Deep Spring, Ash Meadows Spring), Amargosa Desert, Nye Co., Nevada $\left(36^{\circ} 22^{\prime} 29^{\prime \prime}\right.$ N Lat, $116^{\circ} 16^{\prime} 26^{\prime \prime} \mathrm{W}$ Long) $31.4 \mathrm{~km}$ SSE of Lathrop Wells, Nevada. The spring discharged $3.9 \mathrm{~m}^{3} / \mathrm{min}$. at $28.5^{\circ} \mathrm{C}$ at alt. $682.8 \mathrm{~m}$ above $\mathrm{msl}$ from valley fill.

\section{DE-670341. Devils Hole}

$27,000 \pm 2800$

Sample coll. $5 \mathrm{~m}$ below water surface Dec. 9, 1966 from Devils Hole, Death Valley National Monument, Nye Co., Nevada (36 25' 32" N Lat, $116^{\circ} 17^{\prime} 27^{\prime \prime}$ W Long) $22.4 \mathrm{~km}$ SSE of Lathrop Wells, Nevada. Water temperature was $32.0^{\circ} \mathrm{C}$ and alt. of water surface was $719.6 \mathrm{~m}$ above msl. 


\section{DE-670342. Spring 17S/50-35cal}

$15,300 \pm 1200$

Sample coll. Dec. 9, 1966 from Spring 17S/50-35cal, Amargosa Desert, Nye Co., Nevada (36 $25^{\prime} 41^{\prime \prime} \mathrm{N}$ Lat, $116^{\circ} 18^{\prime} 35^{\prime \prime} \mathrm{W}$ Long) $1.6 \mathrm{~km} \mathrm{~W}$ of Devils Hole, Death Valley National Monument. Spring discharges at $31.0^{\circ} \mathrm{C}$ at alt. $743.7 \mathrm{~m}$ above msl from valley fill.

\section{DE-670343. Cactus Spring}

$13,700 \pm 600$

Sample coll. Dec. 10, 1966 from Cactus Spring at Cactus Springs, Clark Co., Nevada $\left(36^{\circ} 33^{\prime} 55^{\prime \prime} \mathrm{N}\right.$ Lat, $115^{\circ} 45^{\prime} 39^{\prime \prime} \mathrm{W}$ Long). The spring when pumped yields $0.02 \mathrm{~m}^{3} / \mathrm{min}$. at $20.5^{\circ} \mathrm{C}$ at alt. $975.4 \mathrm{~m}$ above msl from lake beds and valley fill.

\section{DE-670344. Corn Creek Springs}

$13,900 \pm 800$

Sample coll. Dec. 11, 1966 from SW area of major discharge in pond $\mathrm{lm}$ below bottom of pond, Corn Creek Springs, Clark Co., Nevada $\left(36^{\circ}\right.$ $26^{\prime} 17^{\prime \prime} \mathrm{N}$ Lat, $115^{\circ} 21^{\prime} 16^{\prime \prime} \mathrm{W}$ Long) $31.4 \mathrm{~km}$ NW of Las Vegas, Nevada. Spring discharges at $21.5^{\circ} \mathrm{C}$ along fault scarp assoc. with Las Vegas shear zone at alt. $890.0 \mathrm{~m}$ above $\mathrm{msl}$ from valley fill.

\section{DE-670345. Cold Creek Spring}

$1500 \pm 1000$

Sample coll. Dec. 12, 1966 from Cold Creek Spring, Spring Mountains, Clark Co., Nevada ( $36^{\circ} 24^{\prime} 41^{\prime \prime} \mathrm{N}$ Lat, $115^{\circ} 44^{\prime} 20^{\prime \prime} \mathrm{W}$ Long) $20.9 \mathrm{~km}$ SSE of Indian Springs, Nevada. The spring discharges $2.6 \mathrm{~m}^{3} / \mathrm{min}$. at $10.0^{\circ} \mathrm{C}$ at alt. $1900 \mathrm{~m}$ above msl from Paleozoic limestone.

\section{DE-670346. Grapevine Spring}

$12,800 \pm 700$

10,850 в.C.

Sample coll. Dec. 13, 1966 from Grapevine Spring near Pahrump, Nye Co., Nevada ( $36^{\circ} 27^{\prime} 26^{\prime \prime} \mathrm{N}$ Lat, $116^{\circ} 01^{\prime} 14^{\prime \prime} \mathrm{W}$ Long) $14.5 \mathrm{~km}$ SE of junction of U.S. Hwy. 95 and Nevada 16. The spring discharges 0.04 $\mathrm{m}^{3} / \mathrm{min}$. at $19.0^{\circ} \mathrm{C}$ at alt. $1450 \mathrm{~m}$ above msl from Paleozoic clastic rocks.

\section{DE-670347. Indian Spring \\ $13,500 \pm 800$}

Sample coll. Dec. 14, 1966 from Indian Spring at Indian Springs, Clark Co., Nevada (36 $34^{\prime} 22^{\prime \prime} \mathrm{N}$ Lat, $115^{\circ} 39^{\prime} 31^{\prime \prime} \mathrm{W}$ Long). The spring discharges $1.5 \mathrm{~m}^{3} / \mathrm{min}$. at $25.0^{\circ} \mathrm{C}$ at alt. $975.4 \mathrm{~m}$ above msl from Paleozoic limestone.

\section{DE-670348. Willow Spring}

$1800 \pm 100$

Sample coll. Dec. 14, 1966 from Willow Spring, Clark Co., Nevada $\left(36^{\circ} 25^{\prime} 01^{\prime \prime} \mathrm{N}\right.$ Lat, $115^{\circ} 45^{\prime} 51^{\prime \prime} \mathrm{W}$ Long) $17.7 \mathrm{~km} \mathrm{SSW}$ of Indian Springs, Nevada. Spring discharges at $10.5^{\circ} \mathrm{C}$ at alt. $1829 \mathrm{~m}$ above msl from valley fill over Paleozoic limestone. 


\section{DE-680076. Well 9A}

$9900 \pm 300$

Sample coll. July 7, 1967 from Well 9A, Tonopah Test Range, Nye Co., Nevada ( $37^{\circ} 50^{\prime} 40^{\prime \prime} \mathrm{N}$ Lat, $116^{\circ} 42^{\prime} 40^{\prime \prime} \mathrm{W}$ Long). Well drilled and cased to depth $83.5 \mathrm{~m}$ in 1962 . Well yields $<0.02 \mathrm{~m}^{3} / \mathrm{min}$. at $17.5^{\circ} \mathrm{C}$ from valley fill. Alt. of 1 sd is $1630.7 \mathrm{~m}$ above $\mathrm{msl}$ and static water level was $44.2 \mathrm{~m}$ below $1 \mathrm{sd}$.

\section{DE-680078. Well No. 7}

$19,200 \pm 900$ 17,250 в.c.

Sample coll. July 7, 1967 from Well No. 7, Tonopah Test Range, Nye Co., Nevada (37 $57^{\prime} 20^{\prime \prime} \mathrm{N}$ Lat, $116^{\circ} 45^{\prime} 00^{\prime \prime}$ W Long). Well drilled and cased to depth $226.5 \mathrm{~m}$ in 1963 . Well yields $0.3 \mathrm{~m}^{3} / \mathrm{min}$. at $25.0^{\circ} \mathrm{C}$ from valley fill. Alt. of lsd is $1682.5 \mathrm{~m}$ above $\mathrm{msl}$ and static water level was $110.0 \mathrm{~m}$ below lsd.

\section{DE-680080. Spring 10S/47-14bab}

$15,500 \pm 500$

Sample coll. July 5, 1967 from Spring 10S/47-14bab, Oasis Valley, Nye Co., Nevada ( $37^{\circ} 04^{\prime} 40^{\prime \prime} \mathrm{N}$ Lat, $116^{\circ} 41^{\prime} 10^{\prime \prime} \mathrm{W}$ Long) $24.1 \mathrm{~km}$ NNW of Beatty, Nevada. The spring discharges $1.3 \mathrm{~m}^{3} / \mathrm{min}$. at $29.0^{\circ} \mathrm{C}$ at alt. $1213.7 \mathrm{~m}$ above $\mathrm{msl}$ from valley fill.

\section{DE-680082. Well 10S/47-30dec2}

$10,800 \pm 300$

Sample coll. July 5, 1967 from Well 10 S/47-30dcc2, Oasis Valley, Nye Co., Nevada $\left(37^{\circ} 02^{\prime} 10^{\prime \prime} \mathrm{N}\right.$ Lat, $116^{\circ} 45^{\prime} 20^{\prime \prime} \mathrm{W}$ Long) $14.5 \mathrm{~km}$ NNW of Beatty, Nevada. Well drilled to $36.9 \mathrm{~m}$ and cased to $19.8 \mathrm{~m}$ in 1939 . Well yields $0.2 \mathrm{~m}^{3} / \mathrm{min}$ at $22.5^{\circ} \mathrm{C}$ from valley fill. Alt. of $1 \mathrm{sd} 1182 \mathrm{~m}$ above $\mathrm{msl}$ and static water level was $8.6 \mathrm{~m}$ below lsd.

\section{DE-680089. Spring 11S/47-3cdbl}

$9600 \pm 400$ Nye Co Nevall July 5, 1967 from Spring 11S/47-3cdbl, Oasis Valley, 的 of Beatty, Nevada. The spring discharges $0.2 \mathrm{~m}^{3} / \mathrm{min}$. at $23.0^{\circ} \mathrm{C}$ at alt. $1170.4 \mathrm{~m}$ above $\mathrm{msl}$ from Tertiary volcanic rocks.

\section{DE-680092. Spring 11S/47-10ceb}

$10,300 \pm 400$

Sample coll. July 6, 1967 from Spring 11S/47-10ccb, Oasis Valley, Nye Co., Nevada (36 59' $30^{\prime \prime}$ N Lat, $116^{\circ} 42^{\prime} 50^{\prime \prime}$ W Long) $9.7 \mathrm{~km} \mathrm{NNE}$ of Beatty, Nevada. The spring discharges $1.8 \mathrm{~m}^{3} / \mathrm{min}$. at $21.0^{\circ} \mathrm{C}$ at alt. $1112.5 \mathrm{~m}$ above msl from valley fill.

\section{DE-680097. Spring 11S/47-21aba2}

$10,900 \pm 300$

Sample coll. July 4, 1967 from Spring 11S/47-21aba2, Oasis Valley, Nye Co., Nevada ( $36^{\circ} 58^{\prime} 20^{\prime \prime} \mathrm{N}$ Lat, $116^{\circ} 42^{\prime} 50^{\prime \prime}$ W Long) $7.2 \mathrm{~km}$ NNE of Beatty, Nevada. The spring discharges $<0.04 \mathrm{~m}^{3} / \mathrm{min}$. at $41.0^{\circ} \mathrm{C}$ at alt. $1097.3 \mathrm{~m}$ above $\mathrm{msl}$ from valley fill. 
DE-680098. Well 11S/47-21acel

$7900 \pm 300$

Sample coll. July 4, 1967 from Well 11S/47-21 accl, Oasis Valley, Nye Co., Nevada $\left(36^{\circ} 58^{\prime} 00^{\prime \prime} \mathrm{N}\right.$ Lat, $116^{\circ} 43^{\prime} 20^{\prime \prime} \mathrm{W}$ Long) $6.4 \mathrm{~km}$ NNE of Beatty, Nevada. Well yields $0.1 \mathrm{~m}^{3} / \mathrm{min}$. at $31.5^{\circ} \mathrm{C}$ from valley fill. Alt. of lsd is $1085 \mathrm{~m}$ above msl and static water level was $3.0 \mathrm{~m}$ below lsd.

\section{DE-680101. Well 11S/47-27cba}

$8100 \pm 200$

Sample coll. July 3, 1967 from Well $11 \mathrm{~S} / 47-27 \mathrm{cba}$, Oasis Valley, Nye Co., Nevada $\left(36^{\circ} 57^{\prime} 00^{\prime \prime} \mathrm{N}\right.$ Lat, $116^{\circ} 42^{\prime} 50^{\prime \prime} \mathrm{W}$ Long) $6.4 \mathrm{~km} \mathrm{NE}$ of Beatty, Nevada. Well drilled and cased to $16.8 \mathrm{~m}$ in 1963 and yields 0.08 $\mathrm{m}^{3} / \mathrm{min}$. at $21.5^{\circ} \mathrm{C}$ from valley fill. Alt. of 1 sd is $1060.7 \mathrm{~m}$ above $\mathrm{msl}$ and static water level was $9.1 \mathrm{~m}$ below $1 \mathrm{sd}$.

\section{0 в.C.}

Sample coll. July 3, 1967 from Beatty Spring (Municipal spring), Oasis Valley, Nye Co., Nevada (36 $56^{\circ} 40^{\prime \prime} \mathrm{N}$ Lat, $116^{\circ} 44^{\prime} 40^{\prime \prime} \mathrm{W}$ Long) $1.6 \mathrm{~km}$ NE of Beatty, Nevada. Spring discharges $1.8 \mathrm{~m}^{3} / \mathrm{min}$. at $24.0^{\circ} \mathrm{C}$ at alt. $1027.2 \mathrm{~m}$ above msl from valley fill.

\section{DE-680106. Well 11S/47-6edd}

$8300 \pm 300$

Sample coll. July 3, 1967 from Well 11S/47-6cdd, Oasis Valley, Nye Co., Nevada ( $36^{\circ} 55^{\prime} 00^{\prime \prime} \mathrm{N}$ Lat, $116^{\circ} 55^{\prime} 50^{\prime \prime} \mathrm{W}$ Long) $0.8 \mathrm{~km}$ NNW of Beatty, Nevada. Well drilled to $54.9 \mathrm{~m}$ and cased to $29.0 \mathrm{~m}$ in 1962 and yields $0.3 \mathrm{~m}^{3} / \mathrm{min}$. at $21.5^{\circ} \mathrm{C}$ from valley fill. Alt. of $1 \mathrm{sd}$ is $1030.2 \mathrm{~m}$ above msl and static water level was $333.8 \mathrm{~m}$ below lsd.

\section{DE-680107. Well 12S/47-7bdb}

$3500 \pm 200$

1550 в.c.

Sample coll. July 3, 1967 from Well 12S/47-7bdb, Oasis Valley, Nye Co., Nevada ( $36^{\circ} 54^{\prime} 20^{\prime \prime}$ N Lat, $116^{\circ} 45^{\prime} 30^{\prime \prime}$ W Long) at Beatty, Nevada. Well drilled to $91.4 \mathrm{~m}$ and cased to $54.9 \mathrm{~m}$ in 1965 and yields $0.9 \mathrm{~m}^{3} / \mathrm{min}$. at $20.0^{\circ} \mathrm{C}$ from valley fill. Alt. of 1 sd is $999.7 \mathrm{~m}$ above msl and static water level was $6.7 \mathrm{~m}$ below $1 \mathrm{sd}$.

\section{DE-680458. Well 22S/5-15daa}

$13,800 \pm 1500$

Sample coll. Sept. 4, 1967 from Well 22S/5-15daa, White Sands Missile Range, Dona Anna Co., New Mexico (32 $24^{\prime} 06^{\prime \prime}$ N Lat, $106^{\circ} 24^{\prime} 54^{\prime \prime}$ $\mathrm{W}$ Long) $2.4 \mathrm{~km} \mathrm{~N}$ and $6.4 \mathrm{~km} \mathrm{E}$ of White Sands, New Mexico. Well drilled to $1833.4 \mathrm{~m}$ and cased to $121.9 \mathrm{~m}$ in 1967 . The interval sampled was between $1792.2 \mathrm{~m}$ and $1795.3 \mathrm{~m}$ below 1 sd. The interval yielded $\approx 0.01$ $\mathrm{m}^{3} / \mathrm{min}$. at $54.5^{\circ} \mathrm{C}$. Alt. of lsd is $1204.0 \mathrm{~m}$ above $\mathrm{msl}$ and the static water level was $59.4 \mathrm{~m}$ below $1 \mathrm{sd}$. 


\section{DE-680583. Well Army-1}

$25,100 \pm 2600$

23,150 в.C.

Sample coll. Aug. 1967 from Well Army-1, Nevada Test Site, Nye Co., Nevada $\left(36^{\circ} 35^{\prime} 30^{\prime \prime} \mathrm{N}\right.$ Lat, $116^{\circ} 02^{\prime} 14^{\prime \prime} \mathrm{W}$ Long) $4.0 \mathrm{~km}$ W of junc. of Mercury Hwy. and U.S. 95 . Well drilled to $593.1 \mathrm{~m}$ and cased to $414.5 \mathrm{~m}$ and yields $1.7 \mathrm{~m}^{3} / \mathrm{min}$. at $30.5^{\circ} \mathrm{C}$ from Paleozoic limestone. Alt. of lsd is $961.3 \mathrm{~m}$ above msl and static water level was $239.3 \mathrm{~m}$ below $1 \mathrm{sd}$.

\section{DE-680649. Well HTH-1}

$5300 \pm 200$

Sample coll. Aug. 25, 1967 from Well HTH-1, Hot Creek Valley, Nye Co., Nevada (38 $27^{\prime} 34.80^{\prime \prime} \mathrm{N}$ Lat, $116^{\circ} 12^{\prime} 45.30^{\prime \prime} \mathrm{W}$ Long) $16.1 \mathrm{~km}$ NE of Hot Creek Ranch, Nevada. Well drilled and cased to $1129.0 \mathrm{~m}$ in 1967 in valley fill. Alt. of 1 sd is $1832 \mathrm{~m}$ above msl and static water level was $170 \mathrm{~m}$ below lsd.

\section{DE-680650. Well SMR-4}

$5900 \pm 300$

Sample coll. Nov. 14, 1967 from Well SMR-4, White Sands Missile Range, Dona Anna Co., New Mexico ( $32^{\circ} 27^{\prime} 38^{\prime \prime} \mathrm{N} \mathrm{Lat,} 106^{\circ} 27^{\prime} 15^{\prime \prime} \mathrm{W}$ Long) $9.7 \mathrm{~km} \mathrm{~N}$ and $2.4 \mathrm{~km} \mathrm{E}$ of White Sands, New Mexico. Well drilled to $137.2 \mathrm{~m}$ in 1967 and yields ca. $0.05 \mathrm{~m}^{3} / \mathrm{min}$. at $24.0^{\circ} \mathrm{C}$ from valley fill. Alt. of lsd is $1283.2 \mathrm{~m}$ above msl and static water level was $83.2 \mathrm{~m}$ below $1 \mathrm{scl}$.

\section{DE-680651. Well SMR-4}

$20,300 \pm 1800$

Sample coll. Nov. 20, 1967 from Well SMR-4, White Sands Missile Range, Dona Anna Co., New Mexico (32 $27^{\prime} 38^{\prime \prime} \mathrm{N}$ Lat, $106^{\circ} 27^{\prime} 15^{\prime \prime} \mathrm{W}$ Long) $9.7 \mathrm{~km} \mathrm{~N}$ and $2.4 \mathrm{~km} \mathrm{E}$ of White Sands, New Mexico. Well drilled to $137.2 \mathrm{~m}$ in 1967 and yields ca. $0.05 \mathrm{~m}^{3} / \mathrm{min}$. from valley fill. Alt. of 1 sd is $1283.2 \mathrm{~m}$ above $\mathrm{msl}$ and static water level was $83.2 \mathrm{~m}$ below $1 \mathrm{sd}$.

\section{DE-680759. Well SMR-5}

$12,000 \pm 600$

Sample coll. Dec. 11, 1967 from Well SMR-5, White Sands Missile Range, Dona Anna Co., New Mexico (32 $28^{\prime} 32^{\prime \prime}$ N Lat, $106^{\circ} 23^{\prime} 50^{\prime \prime} \mathrm{W}$ Long) $11.3 \mathrm{~km} \mathrm{~N}$ and $8.0 \mathrm{~km} \mathrm{E}$ of White Sands, New Mexico. Well drilled and cased to $75.9 \mathrm{~m}$ in 1967 and yields $0.08 \mathrm{~m}^{3} / \mathrm{min}$. at $21.0^{\circ} \mathrm{C}$ from valley fill. Alt. of lsd is $1204.0 \mathrm{~m}$ above msl and static water level was $33.1 \mathrm{~m}$ below lsd.

\section{DE-680773. Constantine Spring}

$3300 \pm 300$

Sample coll. Sept. 27, 1967 from Constantine Spring, Amchitka I., Alaska (51 $22^{\prime} 43^{\prime \prime} \mathrm{N}$ Lat, $179^{\circ} 14^{\prime} 59^{\prime \prime} \mathrm{E}$ Long) $0.8 \mathrm{~km}$ N of Base Camp, Amchitka I., Alaska. The spring discharges $0.73 \mathrm{~m}^{3} / \mathrm{min}$. at $30.0^{\circ} \mathrm{C}$ at alt. $27.7 \mathrm{~m}$ above msl from Pleistocene gravel. 


\section{DE-680782. Well Skelly Jal WSW-1}

Sample coll. Oct. 3, 1967 from Well Skelly Jal WSW-1, Lea Co., New Mexico $\left(32^{\circ} 12^{\prime} 30^{\prime \prime} \mathrm{N}\right.$ Lat, $103^{\circ} 16^{\prime} 25^{\prime \prime} \mathrm{W}$ Long) $9.7 \mathrm{~km} \mathrm{~N}$ and $8.0 \mathrm{~km} \mathrm{~W}$ of Jal. Well drilled to $1371.6 \mathrm{~m}$ and cased to $1204.3 \mathrm{~m}$ in 1967 . Alt. is $1030.2 \mathrm{~m}$ above $\mathrm{msl}$ and static water level is $1205.5 \mathrm{~m}$ below $1 \mathrm{sd}$.

\section{DE-680788. Well}

$9900 \pm 700$

7950 B.C.

Sample coll. Oct. 3, 1967 from well, White Sands Missile Range, Dona Anna Co., New Mexico $2.4 \mathrm{~km} \mathrm{~N}$ and $6.4 \mathrm{~km} \mathrm{E}$ of White Sands, New Mexico. Well drilled to $1833.4 \mathrm{~m}$ and cased to $121.9 \mathrm{~m}$ in 1967 and yields $0.002 \mathrm{~m}^{3} / \mathrm{min}$. at $53.5^{\circ} \mathrm{C}$ from lakebed sediments. Alt. is $1204.0 \mathrm{~m}$ above msl and static water level was $119 \mathrm{~m}$ below $1 \mathrm{sd}$.

\section{DE-680905. Base Camp Well}

$$
\begin{aligned}
& 10,500 \pm 400 \\
& 8550 \text { в.с. }
\end{aligned}
$$

Sample coll. Feb. 14, 1968 from Base camp Well, Hot Creek Valley, Nye Co., Nevada ( $38^{\circ} 18^{\prime} 20^{\prime \prime} \mathrm{N}$ Lat, $116^{\circ} 16^{\prime} 55^{\prime \prime} \mathrm{W}$ Long) $16.1 \mathrm{~km} \mathrm{E}$ of Warm Springs, Nevada at USAEC CNTA Base Camp. Well drilled and cased to $90.5 \mathrm{~m}$ below lsd in 1967 and yielded $>0.2 \mathrm{~m}^{3} / \mathrm{min}$. at $17.0^{\circ} \mathrm{C}$ from valley fill. Alt. of lsd is $585 \mathrm{~m}$ above $\mathrm{msl}$ and static water level was $24.4 \mathrm{~m}$ below lsd.

\section{DE-680914. Amargosa Tracer Well No. 2}

Sample coll. Feb. 1968 from Amargosa Tracer Well No. 2, Amargosa Valley, Nye Co., Nevada (36 $32^{\prime} 11^{\prime \prime}$ N Lat, $116^{\circ} 13^{\prime} 39^{\prime \prime}$ W Long) $27.4 \mathrm{~km}$ SW of Mercury, Nevada. Well drilled to $252.4 \mathrm{~m}$ and cased to $200.9 \mathrm{~m}$ below lsd in 1966 and yields $3.4 \mathrm{~m}^{3} / \mathrm{min}$. at $31.0^{\circ} \mathrm{C}$. Alt. of $1 \mathrm{sd}$ is $731.6 \mathrm{~m}$. above msl and static water level was $11.9 \mathrm{~m}$ below $1 \mathrm{sd}$.

\section{DE-680960. Well C}

$22,900 \pm 1200$

\section{0,950 в.C.}

Sample coll. Mar. 4, 1968 from Well C, Yucca Flat, Nevada Test Site, Nye Co.. Nevada ( $36^{\circ} 55^{\prime} 08^{\prime \prime} \mathrm{N}$ Lat, $116^{\circ} 00^{\prime} 35^{\prime \prime}$ W Long) $30 \mathrm{~km} \mathrm{~N}$ of Mercury, Nevada. Well drilled and cased to $518.5 \mathrm{~m}$ below lsd in 1961 and yields 0.8 to $1.7 \mathrm{~m}^{3} / \mathrm{min}$. at $36.5^{\circ} \mathrm{C}$ from Paleozoic limestone. Alt. of lsd is $1195.1 \mathrm{~m}$ above $\mathrm{msl}$ and static water level was $469.4 \mathrm{~m}$ below $1 \mathrm{sd}$.

TABle 3.

Summary of $\mathrm{C}^{14}, \delta \mathrm{C}^{13}$, and alkalinity for water sources in Alaska

\begin{tabular}{ccccc}
\hline $\begin{array}{c}\text { Sample } \\
\text { no. }\end{array}$ & Date & $\begin{array}{c}\text { Radiocarbon } \\
(\% \mathrm{NBS})\end{array}$ & $\begin{array}{c}\delta \mathrm{C}^{13} \\
(\% \mathrm{PDB})\end{array}$ & $\begin{array}{c}\text { Total alkalinity } \\
\text { as bicarbonate } \\
(\mathrm{mg} / \mathrm{l})\end{array}$ \\
\hline $\mathrm{DE}-680773$ & $09 / 27 / 67$ & $66.6 \pm 2.5$ & -16.6 & 92 \\
\hline
\end{tabular}




\section{DE-680961. Well C}

20,750 в.c.

Sample coll. Mar. 5, 1968 from Well C, Yucca Flat, Nevada Test Site, Nye Co., Nevada ( $36^{\circ} 55^{\prime} 08^{\prime \prime} \mathrm{N}$ Lat, $116^{\circ} 00^{\prime} 35^{\prime \prime} \mathrm{W}$ Long) $30 \mathrm{~km} \mathrm{~N}$ of Mercury, Nevada.

\section{DE-680962. Well C}

$20,700 \pm 900$

Sample coll. Mar. 6, 1968 from Well C, Yucca Flat, Nevada Test Site, Nye Co., Nevada $\left(36^{\circ} 55^{\prime} 08^{\prime \prime} \mathrm{N}\right.$ Lat, $116^{\circ} 00^{\prime} 35^{\prime \prime} \mathrm{W}$ Long) $30 \mathrm{~km} \mathrm{~N}$ of Mercury, Nevada.

\section{DE-680963. Well C}

$17,100 \pm 800$

Sample coll. Mar. 7, 1968 from Well C, Yucca Flat, Nevada Test Site, Nye Co., Nevada (36 $56^{\circ} 08^{\prime \prime} \mathrm{N}$ Lat, $116^{\circ} 00^{\prime} 35^{\prime \prime}$ W Long) $30 \mathrm{~km} \mathrm{~N}$ of Mercury, Nevada.

\section{DE-680964. Well C}

Sample coll. Mar. 8, 1968 from Well C, Yucca Flat, Nevada Test Site, Nye Co., Nevada (36 $55^{\circ} 08^{\prime \prime} \mathrm{N}$ Lat, $116^{\circ} 00^{\prime} 35^{\prime \prime} \mathrm{W}$ Long) $30 \mathrm{~km} \mathrm{~N}$ of Mercury, Nevada.

\section{DE-681240. Clover Creek}

Sample coll. June 18, 1968 from Clover Creek, Little Fish Lake Valley, Nye Co., Nevada (38 $40^{\prime} 40^{\prime \prime} \mathrm{N}$ Lat, $116^{\circ} 29^{\prime} 30^{\prime \prime} \mathrm{W}$ Long) $8.0 \mathrm{~km}$ SW of Fish Lake Ranch, Nevada at alt. of $1975 \mathrm{~m}$ above msl.

\section{REFERENCES}

Buckley, J. D. and Willis, E. H., 1969, Isotopes' radiocarbon measurements VII: Radiocarbon, v. 11, p. 53-105.

Craig, Harmon, 1957, Isotopic standards for carbon and oxygen and correction factors for mass-spectrometric analysis of carbon dioxide: Geochim. et Cosmochim. Acta, v. 12, p. 133-149.

Noakes, J. E., Kim, S. M., and Akers, L. K., 1967, Recent improvements in benzene chemistry for radiocarbon counting: Geochim. et Cosmochim. Acta, v. 31, p. 10941096.

Rainwater, F. H. and Thatcher, L. L., 1960, Methods for collection and analysis of water samples: U.S. Geol. Survey Water-Supply Paper 1454, p. 94-95. 